\title{
Mise au point d'une grille de notation de l'état corporel des ânes de trait au Nord Cameroun
}

\author{
E. Vall ${ }^{1}$ A.L. Ebangi ${ }^{2}$ O. Abakar ${ }^{3}$
}

\begin{abstract}
Mots-clés
Ane - Etat corporel - Evaluation Surveillance nutritionnelle - Energie animale - Cameroun.
\end{abstract}

\begin{abstract}
Résumé
Apparue dans les années 1970 au Nord Cameroun, la traction asine est aujourd'hui utilisée par 25000 "petits » agriculteurs. La capacité de travail de l'âne est juste suffisante pour les travaux agricoles. Le maintien dans un état nutritionnel correct est déterminant pour garantir une endurance à l'effort acceptable. Cette condition est loin d'être satisfaite. Dans cette étude, l'état nutritionnel des ânes de trait du Nord Cameroun a été caractérisé au moyen d'une grille de notation de l'état corporel. Une note de dos et une note de flanc, sur une échelle de 1 à 4 (émacié, maigre, moyen, bon) ont été attribuées à vue selon l'aspect du bassin, de la colonne vertébrale et du côté. La moyenne des deux notes, arrondie au demi-point supérieur, a donné la note globale. La corrélation des notes entre différents notateurs a été voisine de 80 p. 100, ce qui est un bon indice de reproductibilité. Un suivi de 41 mâles et 34 femelles a montré que durant la saison sèche, la note 3 a largement dominé (50 à 70 p. 100). D'octobre à janvier, les animaux maigres (note 2), ayant profité du changement de saison et d'environnement pathologique, ont reconstitué leurs réserves, tandis que les animaux bons (note 4) ont maigri avec les premières rigueurs de la saison sèche. De janvier à juin, la raréfaction progressive des ressources alimentaires a provoqué un amaigrissement généralisé, femelles gravides exceptées. Chez les mâles, la proportion des notes 2 est passée de 10 à 20 p. 100 entre le début et la fin de la saison sèche. Chez les femelles, cette proportion a été importante et constante tout au long de I'année (30 à 40 p. 100). Des actions d'amélioration de I'alimentation doivent être ciblées sur les femelles.
\end{abstract}

\section{INTRODUCTION}

Au Nord Cameroun, les agriculteurs ont largement adopté la traction asine. On compte aujourd'hui environ 25000 ânes de trait, ce qui représente 30 p. 100 des attelages (7). Cette pratique s'est développée à partir de 1970 dans l'Extrême Nord, notamment sous l'impulsion des missions catholiques, en relais et en complément de la traction bovine. Aujourd'hui, comme l'indique la figure 1, près de 80 p. 100 des attelages asins sont localisés dans les régions au sud de Maroua, et près de Guider et de Kaélé (9). La densité de

1. Cirad-emvt, TA 30/A, Campus international de Baillarguet, 34398 Montpellier Cedex 5, France

2. Irad, Centre de Wakwa, BP 65, Wakwa, Cameroun

3. Irad, Station polyvalente, BP 415, Garoua, Cameroun la population est très élevée dans l'Extrême Nord du Cameroun. La pression foncière sur les terres agricoles est très forte et de nombreux jeunes continuent à s'installer comme agriculteurs, ce qui conduit à la formation d'exploitations de petites dimensions. Une telle concentration d'attelages légers dans cette région s'explique ici par la petite taille moyenne des exploitations, là par la nature caillouteuse des sols sur lesquels il est plus facile de conduire des équipements agricoles de dimensions réduites (charrue 7") et souvent par la coexistence des deux facteurs. Ainsi, pour un nombre important d'agriculteurs modestes, l'âne constitue une solution adaptée à leur besoin d'énergie agricole. Les paysans utilisent indifféremment les mâles et les femelles car leurs forces de traction sont comparables. Cependant, en valeur absolue cette force reste très inférieure à celles des autres espèces de trait et tout juste suffisante pour effectuer l'ensemble des travaux agricoles sur une exploitation de taille modeste (8). 


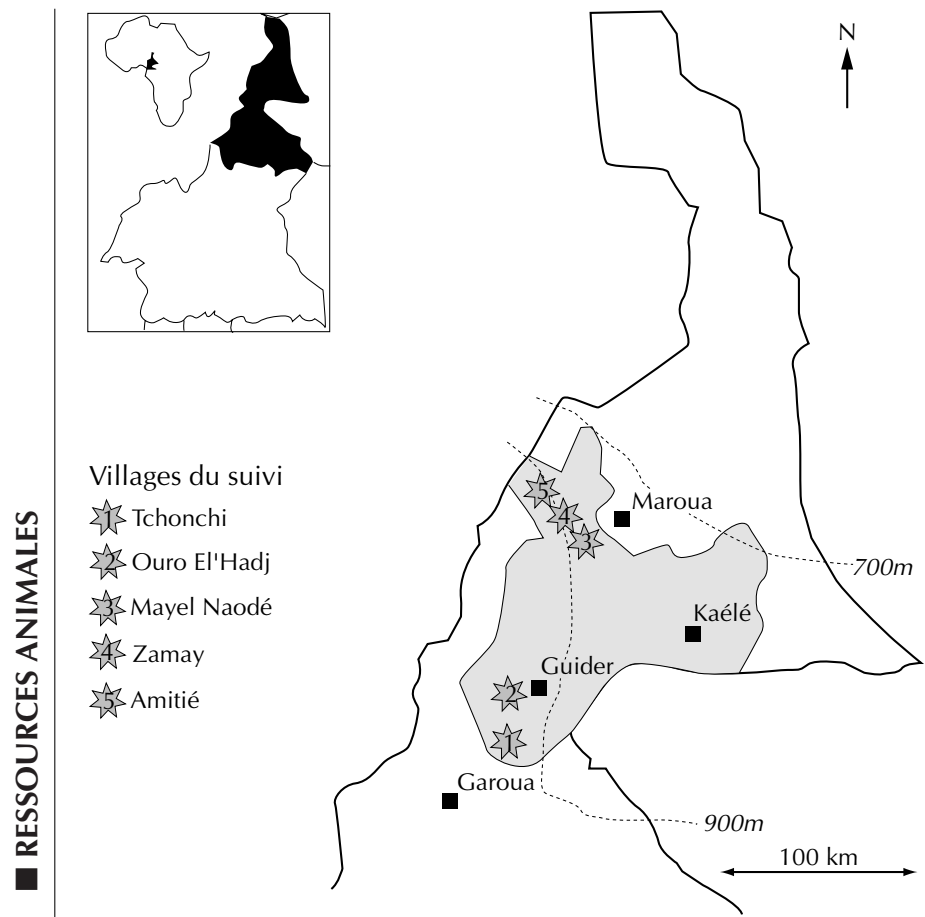

Figure 1 : carte de l'Extrême Nord du Cameroun, localisation des villages du suivi et limites de la région à forte concentration d'ânes de trait.

La capacité de travail d'un animal de trait recouvre deux aspects. Premièrement, la puissance instantanée de l'individu qui est fortement corrélée à son poids vif et à sa masse musculaire. Deuxièmement, l'endurance à l'effort qui dépend surtout de l'état de forme physique de l'animal et tout particulièrement de son état nutritionnel. Dans la région de l'étude, les ânes sont utilisés pour des travaux dont l'intensité dépasse légèrement leur capacité optimale ce qui, sur une courte durée, ne handicape pas l'animal. Le labour et le sarclage requièrent une force de traction équivalente à $20-25$ p. 100 du poids vif de l'âne, pour une capacité optimale de travail estimée à 15 p. 100 du poids vif chez cet animal (8). Cependant, à certaines périodes de l'année, au demeurant déterminantes pour l'agriculteur comme les labours, les attelages sont sollicités très fréquemment, qui plus est sur de longues périodes (au minimum 4 heures par jour, 20 à 30 jours de suite). Dans ces conditions, la résistance à l'effort de longue durée devient un critère déterminant pour l'agriculteur, surtout si celui-ci ne possède qu'un seul animal.

L'âne est une espèce bien adaptée aux conditions difficiles et au climat aride de l'Extrême Nord du Cameroun. Sa ration consiste en aliments simples et peu recherchés qu'il consomme néanmoins en grandes quantités. Malgré cette rusticité avérée, les auteurs ont rencontré dans les villages de la région des ânes de trait présentant un état corporel désolant (maigreur, poils piqués, blessures...) dans une proportion non négligeable. Certains agriculteurs déclarent apporter épisodiquement un complément alimentaire à leur animal (son, tourteau, drêche) et leur réserver un peu de fourrage (9). Manifestement, ces pratiques restent peu suivies et peu répandues. Faisant état de cette situation aux zootechniciens du service élevage de la Société de développement du coton du Cameroun (Sodécoton), ces derniers ont demandé à la recherche d'établir un outil permettant d'estimer simplement l'état nutritionnel des ânes de trait pour identifier les stades critiques nécessitant une adaptation rapide des pratiques d'alimentation ${ }^{*}$.

\footnotetext{
* Parallèlement, les auteurs ont conduit des travaux sur l'adaptation du rationnement de l'âne à l'intensité de l'effort de traction requis. Ces travaux feront l'objet
} d'une autre communication
En 2000, Pearson et Ouassat ont publié une table d'état corporel des ânes de trait comportant neuf niveaux (5). Ces auteurs ont construit leur table à partir d'observations effectuées sur 500 ânes marocains et 60 ânes écossais. Leur objectif a été de dresser une table générale utilisable dans les zones tempérées comme dans les zones tropicales. La présente étude, conduite dans la partie soudano-sahélienne du Cameroun, doit permettre d'élargir le domaine de validité du travail de Pearson et Ouassat. Par ailleurs, le système de la présente étude a été simplifié en proposant une notation à vue sans palpation et une focalisation des observations sur un ensemble de points limités et bien définis. La table ainsi obtenue a été testée sur un échantillon d'ânes suivis durant une saison sèche complète. Les données collectées ont été analysées de façon diachronique pour caractériser les variations de l'état nutritionnel des ânes de trait du Nord Cameroun.

\section{MATERIEL ET METHODES}

\section{Description des ânes dans l'aire de l'étude}

Les caractéristiques des ânes du Nord Cameroun ont fait l'objet de plusieurs études $(1,9,10)$. Ils se rapprochent du type nubien. Comme Equus asinus nubicus, la robe est généralement grise, marquée d'une bande cruciale noire sur l'épaule. Cependant, le gabarit est inférieur au type nubien nominal : en moyenne 130 contre $200 \mathrm{~kg}$ de poids vif et 100 contre $125 \mathrm{~cm}$ de hauteur au garrot (6). Dressés dès l'âge de quatre ans, ils travaillent 10 années et parfois davantage. Une inspection sanitaire effectuée en saison sèche sur 135 individus a révélé un état sanitaire assez satisfaisant (10) ; les principales conclusions de l'étude sont rappelées ci-après. Les accidents de la route sont une cause de mortalité importante, plus que les maladies. Le taux d'hématocrite moyen s'élève à 31 p. 100 . On note cependant une forte proportion de femelles anémiées (20 p. 100 contre 5 p. 100 chez les mâles). Les strongles sont les principaux parasites intestinaux identifiés (40 p. 100 des individus portent plus de 500 œufs/g de fèces). Les ânes sont moins infestés de tiques que les bovins, Amblyoma variegatum et Hyaloma étant les deux principales identifiées. Ils sont souvent porteurs de plaies occasionnées par des harnais mal ajustés, fabriqués avec des matériaux inadéquats (Nylon, caoutchouc).

\section{Echantillonnage}

Un échantillon d'ânes composé de 75 mâles et de 60 femelles, appartenant à des producteurs et répartis dans cinq villages des régions de Guider et du sud de Maroua (figure 1), a été noté à quatre reprises durant la saison sèche de 1996-97. Les sites ont été choisis dans les régions où beaucoup d'agriculteurs utilisent des ânes de trait. Les agriculteurs ont été contactés par les zootechniciens de la Sodécoton. A chaque passage dans un village, le zootechnicien a été présent et a participé aux observations. Pour faciliter la reconnaissance, tous les ânes ont été identifiés.

\section{Collecte des données}

A chaque passage dans un village, l'état corporel des ânes présentés a fait l'objet d'une notation. Les auteurs n'ont disposé au préalable d'aucun référentiel, Pearson et Ouassat n'ayant pas encore publié leurs résultats. Ils ont donc demandé aux paysans de leur décrire les différents niveaux d'embonpoint de leurs ânes de trait. Leurs réponses ont fait état d'une distinction entre deux cas : le «bon animal» et «l'animal maigre ». Ces catégories manifestement trop frustres pour construire une table d'état corporel a conduit les auteurs à nuancer cette représentation. Parmi les ânes dits « bons » ont été distingués un niveau supérieur (obèse) et un niveau inférieur (moyen), et parmi les « maigres », un niveau supé- 
rieur (moyen) et un niveau inférieur (émacié). Cet exercice a conduit à la définition de cinq notes : obèse (5), bon (4), moyen (3), maigre (2) et émacié (1). Il restait à caractériser chaque note.

La technique utilisée a été adaptée du système de l'Iteb mis au point en France sur les vaches Pie noir et qui comporte six notes de 0 à 5 (3). Lors des tournées préparatoires du suivi, les observations faites ont conduit les auteurs à retenir, comme dans la table de l'Iteb, deux régions d'observation sur le corps de l'animal, l'arrière-train et le flanc. Deux notes ont ainsi été attribuées à l'animal. La première (note de dos) a porté sur l'aspect du bassin et de la base de la queue. La seconde (note de flanc) a porté sur le côté au niveau des côtes, du bassin et de la colonne vertébrale. La moyenne des deux notes, arrondie au demi-point supérieur, donne la note globale de l'animal. Ces régions d'observation ont également été retenues par Pearson et Ouassat (5), ce qui a conforté les auteurs dans leur choix à défaut d'autres références disponibles. L'âne a été noté à distance $(2$ à $3 \mathrm{~m})$ sur des critères visuels. Les maniements n'ont pas été indispensables pour l'attribution d'une note. Ils ont permis cependant de conforter le notateur dans son jugement.

\section{Analyse des données}

L'analyse des données s'est basée sur les dates d'observation suivantes : octobre 1996, janvier, avril et juin 1997. Un certain nombre de sorties accidentelles d'animaux (8 cas de mortalité, 3 vols, 2 fractures invalidantes), de ventes (3), de mises à l'écart d'animaux n'ayant pas atteint leur taille adulte (moins de quatre ans), et l'absentéisme d'un certain nombre d'agriculteurs a conduit les auteurs à réduire l'analyse à 75 individus adultes présents aux quatre passages (tableau I).

La table d'état corporel se présente sous la forme de dessins représentant pour chaque note un individu mâle de dos et de flanc. Les dessins ont été effectués à partir de nombreux clichés photographiques tirés du suivi, desquels un portrait-robot a été extrait pour chaque note.

La reproductibilité du système de notation (faculté à produire des notes comparables pour différents notateurs notant le même animal séparément) a été testée au mois de juin 2001 par quatre notateurs (dont trois étaient bien rodés à l'usage de la grille) sur un échantillon de 76 individus. Ce test a permis de calculer des coefficients de corrélation entre notateurs et de produire une analyse de variance entre notateurs.

Les données collectées ont permis d'analyser de façon diachronique : i) les proportions des différentes notes ; ii) les variations des notes selon le sexe pour des groupes partageant la même note de départ.

\section{RESULTATS}

\section{Présentation de la grille}

Les profils arrière et profils de flanc ont été illustrés note par note respectivement sur les figures 2 et 3 . Les critères de notation ont été repris dans le tableau II. La note de dos a été basée sur quatre points anatomiques : i) la pointe de la fesse (tubérosité ischiatique ou partie postérieure du bassin) ; ii) le ligament sacro-tubéral (il relie le sacrum à la tubérosité ischiatique) ; iii) le détroit caudal (espace centré sur l'anus et délimité par les deux ligaments sacrotubéraux, la queue et le plafond du bassin) ; iv) la ligne du dos (colonne vertébrale). La note de flanc a été basée sur les quatre points anatomiques suivants : i) la pointe de la hanche (pointe de l'ilium ou partie antérieure du bassin) ; ii) le creux du flanc ; iii) l'épine dorsale (partie supérieure de la colonne vertébrale, constituée par la succession des apophyses épineuses recouvertes par d'épais ligaments); iv) les côtes.

En définitive, sur l'ensemble des cas observés quatre notes ont été identifiées. L'état d'obésité n'a jamais été rencontré au Nord Cameroun et de fait n'a pas été présenté. Pearson et Ouassat l'ont présenté pour des ânes européens vivant dans des zones tempérées (5). La note 3 a été la plus représentée (50-70 p. 100 des cas selon l'époque), suivie de la note 2 (20 à 30 p. 100 des cas), puis de la note 4 (5 à 25 p. 100) (figure 4). Deux périodes ont pu être distinguées (figure 4) : d'octobre à janvier, les notes 3 ont progressé au détriment des notes 4 , avec les premières rigueurs de la saison sèche ; de janvier à juin, les mauvaises notes (notes 2) ont progressé, passant de 20 à 30 p. 100, et les bonnes aussi (notes 4), allant de 5 à 15 p. 100. La progression des notes 4 a surtout concerné les femelles approchant de la fin de la gestation. L'augmentation des notes 2 s'est expliquée simplement par la raréfaction des ressources alimentaires en fin de saison sèche.

Tableau I

Nombre d'ânes suivis par village

\begin{tabular}{lcccc} 
Villages & Sexe & $\begin{array}{c}\text { Nb. d'ânes } \\
\text { retenus }\end{array}$ & $\begin{array}{c}\text { Nb. d'ânes } \\
\text { bouclés }\end{array}$ & $\begin{array}{c}\text { Anes retenus } \\
\text { par village }(\%)\end{array}$ \\
\hline Tchonchi & M & 12 & 20 & 63 \\
Ouro El Had'j & F & 7 & 10 & 43 \\
Mayel Naodé & F & 10 & 20 & 53 \\
Zamay & M & 3 & 10 & 21 \\
Village amitié & M & 11 & 9 & 60 \\
Total & F & 5 & 17 & 13 \\
\end{tabular}

M : mâle ; F : femelle 

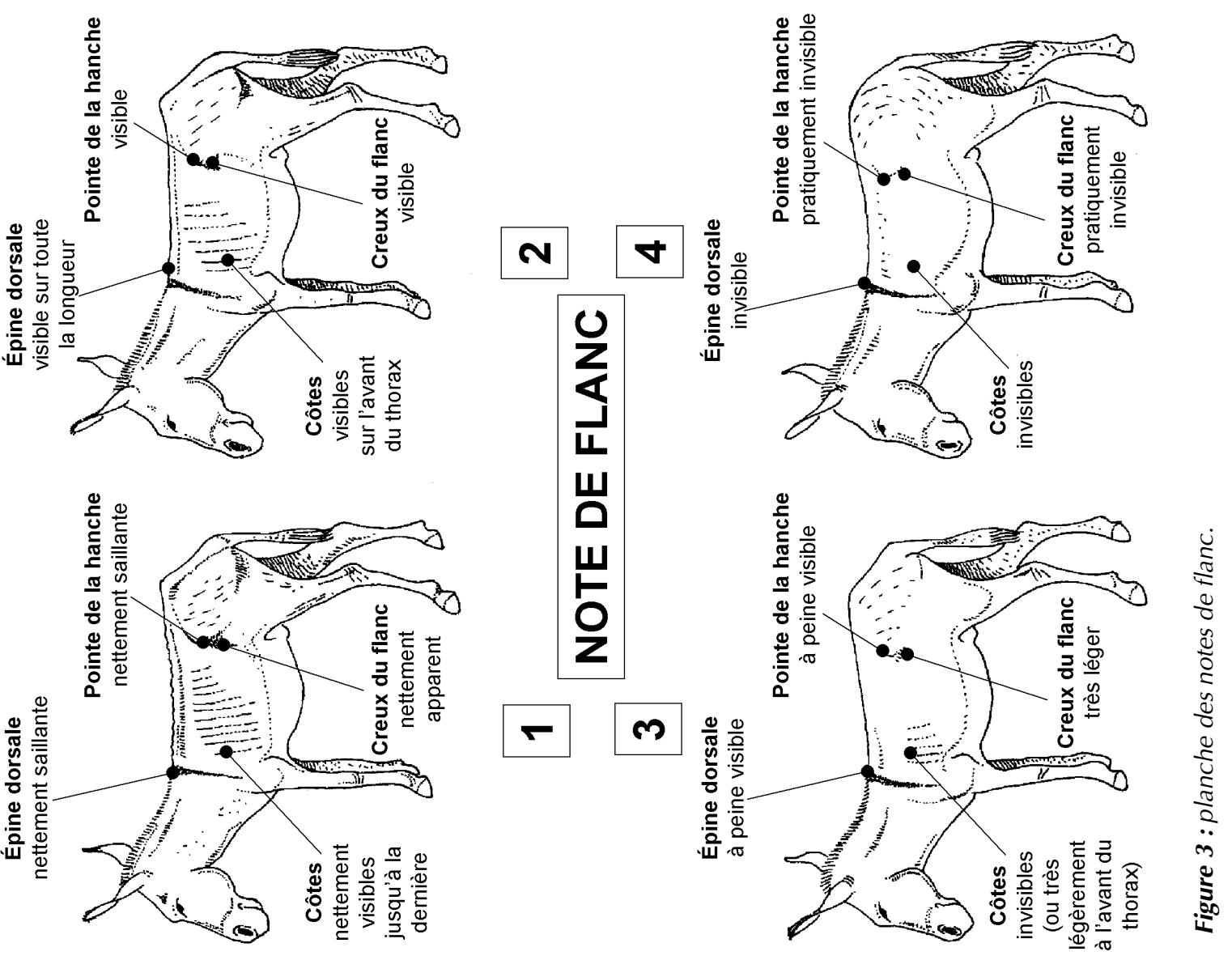

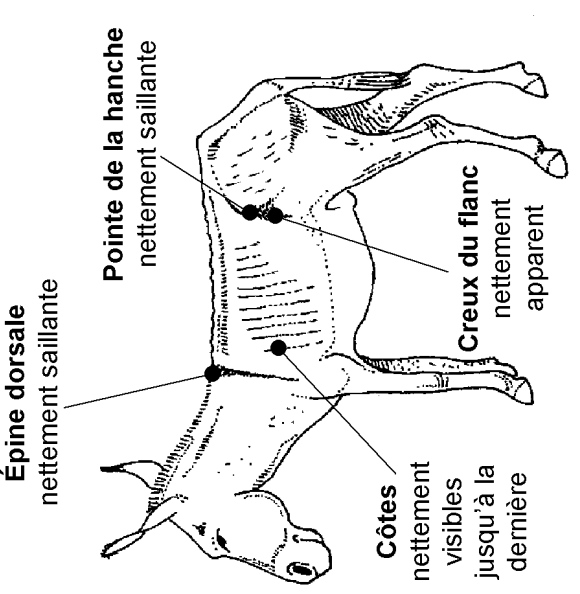

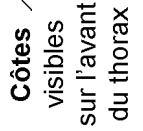

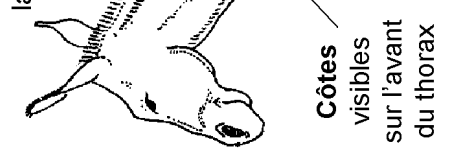

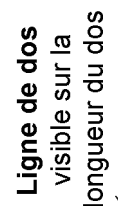
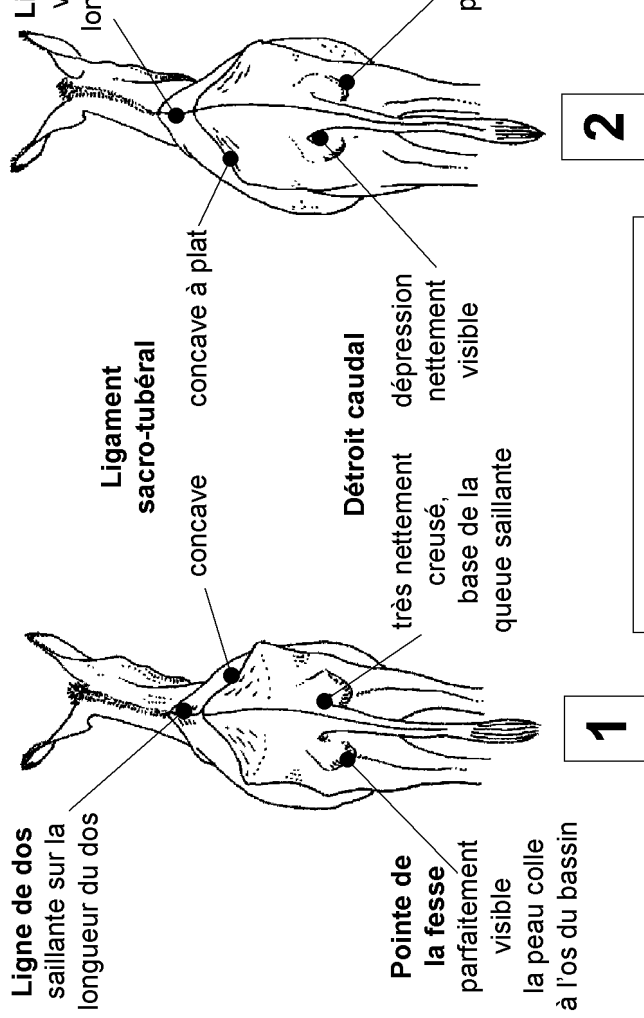
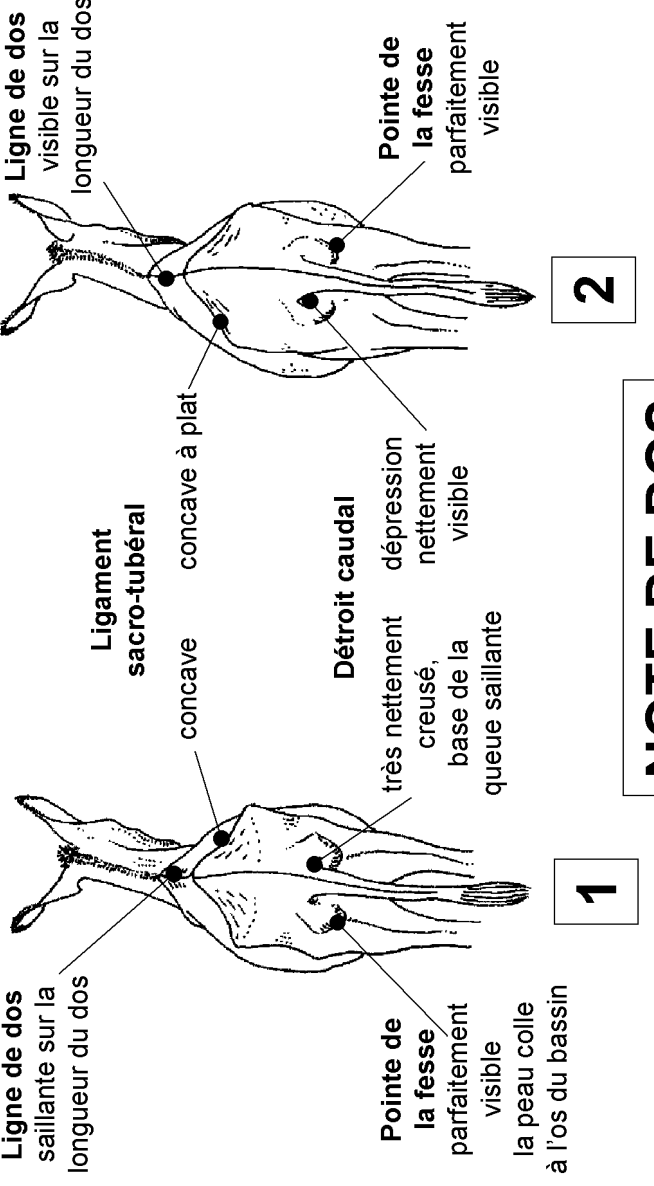

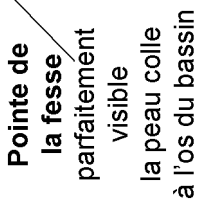

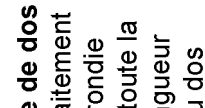

票密

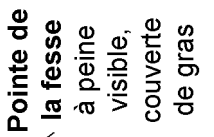

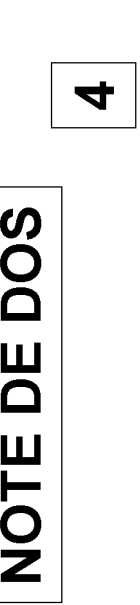

$m$
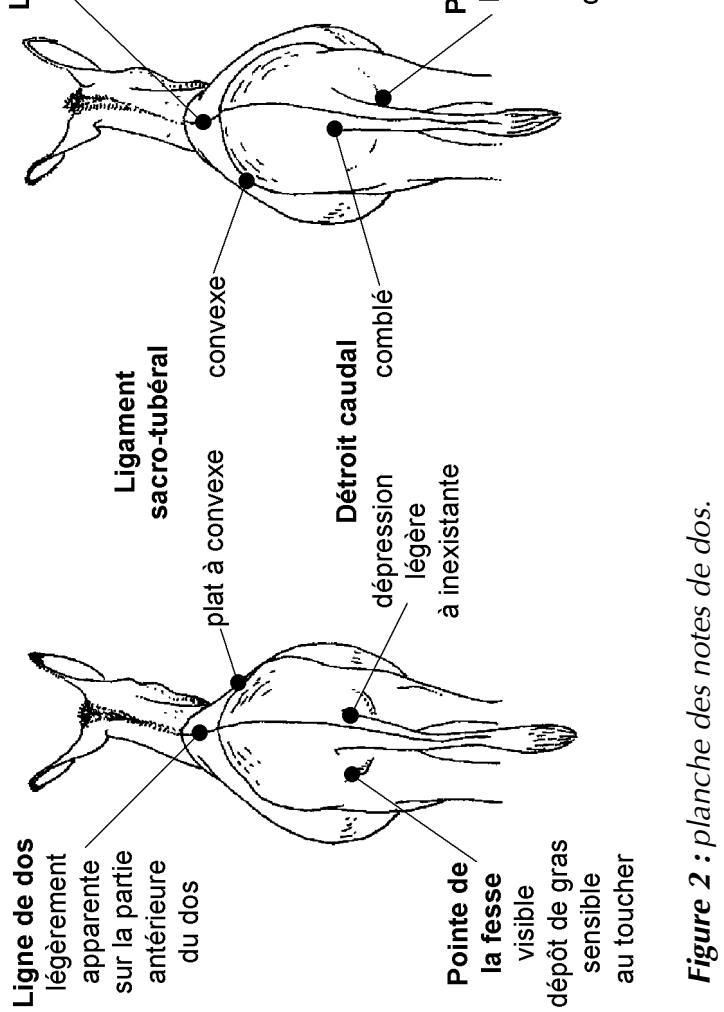


\section{Tableau II}

Critères de notation de l'état corporel d'un âne

\begin{tabular}{|c|c|c|c|c|c|c|c|c|}
\hline \multirow[t]{2}{*}{ Note } & \multicolumn{4}{|c|}{ Note de dos } & \multicolumn{4}{|c|}{ Note de flanc } \\
\hline & $\begin{array}{l}\text { Pointe de } \\
\text { la fesse }\end{array}$ & $\begin{array}{c}\text { Ligament } \\
\text { sacro-tubéral }\end{array}$ & $\begin{array}{l}\text { Détroit } \\
\text { caudal }\end{array}$ & $\begin{array}{l}\text { Ligne } \\
\text { de dos }\end{array}$ & $\begin{array}{l}\text { Pointe de } \\
\text { la hanche }\end{array}$ & $\begin{array}{l}\text { Creux } \\
\text { du flanc }\end{array}$ & $\begin{array}{c}\text { Epine } \\
\text { dorsale }\end{array}$ & Côtes \\
\hline 4 & $\begin{array}{l}\text { A peine } \\
\text { visible, } \\
\text { couverte } \\
\text { de gras }\end{array}$ & Convexe & Comblé & $\begin{array}{l}\text { Parfaitement } \\
\text { arrondie sur } \\
\text { toute la } \\
\text { longueur }\end{array}$ & $\begin{array}{l}\text { Pratiquement } \\
\text { invisible }\end{array}$ & $\begin{array}{l}\text { Pratiquement } \\
\text { invisible }\end{array}$ & invisible & Invisibles \\
\hline 3 & $\begin{array}{l}\text { Visible, dépôt } \\
\text { de gras } \\
\text { sensible } \\
\text { au toucher }\end{array}$ & $\begin{array}{l}\text { Plat } \\
\text { à convexe }\end{array}$ & $\begin{array}{l}\text { Dépression } \\
\text { légère à } \\
\text { inexistante }\end{array}$ & $\begin{array}{l}\text { Légèrement } \\
\text { apparente } \\
\text { sur la partie } \\
\text { antérieure } \\
\text { du dos }\end{array}$ & $\begin{array}{l}\text { A peine } \\
\text { visible }\end{array}$ & Très léger & $\begin{array}{l}\text { A peine } \\
\text { visible }\end{array}$ & $\begin{array}{l}\text { Invisibles } \\
\text { (ou à peine } \\
\text { visible } \\
\text { à l'avant } \\
\text { du thorax) }\end{array}$ \\
\hline 2 & $\begin{array}{l}\text { Parfaitement } \\
\text { visible }\end{array}$ & $\begin{array}{l}\text { Concave } \\
\text { à plat }\end{array}$ & $\begin{array}{l}\text { Dépression } \\
\text { nettement } \\
\text { visible }\end{array}$ & $\begin{array}{l}\text { Visible sur } \\
\text { la longueur } \\
\text { du dos }\end{array}$ & Visible & Visible & $\begin{array}{l}\text { Visible } \\
\text { sur toute } \\
\text { la longueur }\end{array}$ & $\begin{array}{l}\text { Visibles sur } \\
\text { I'avant du } \\
\text { thorax }\end{array}$ \\
\hline 1 & $\begin{array}{l}\text { Parfaitement } \\
\text { visible, la } \\
\text { peau colle } \\
\text { au bassin }\end{array}$ & Concave & $\begin{array}{l}\text { Très } \\
\text { nettement } \\
\text { creusé, base } \\
\text { de la queue } \\
\text { saillante }\end{array}$ & $\begin{array}{l}\text { Saillante sur } \\
\text { la longueur } \\
\text { du dos }\end{array}$ & $\begin{array}{l}\text { Nettement } \\
\text { saillante }\end{array}$ & $\begin{array}{l}\text { Nettement } \\
\text { apparent }\end{array}$ & $\begin{array}{l}\text { Nettement } \\
\text { saillante }\end{array}$ & $\begin{array}{l}\text { Nettement } \\
\text { visibles, } \\
\text { jusqu'à } \\
\text { la dernière }\end{array}$ \\
\hline
\end{tabular}

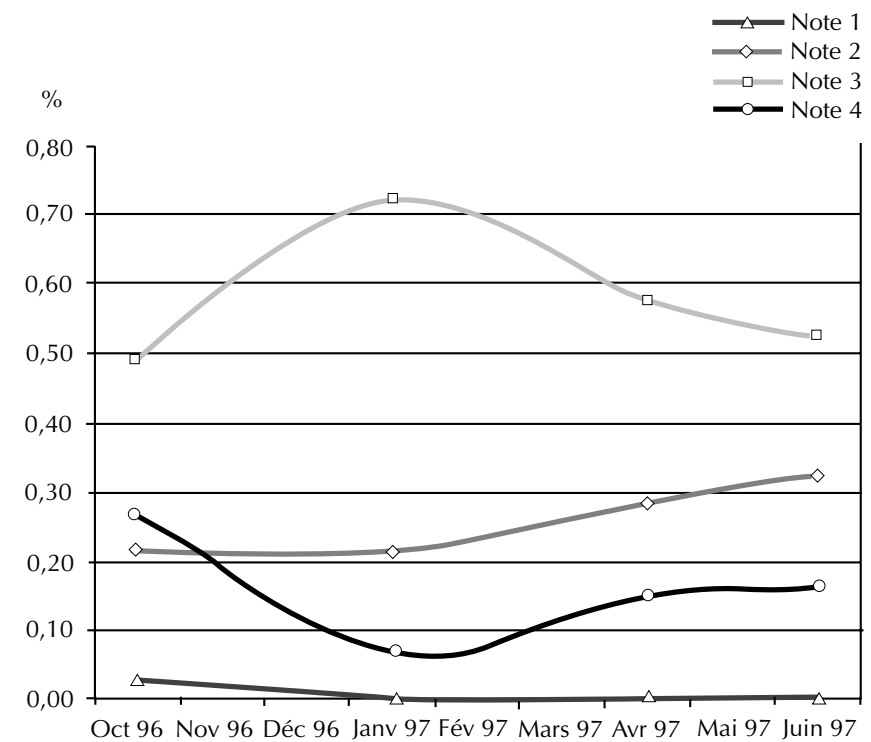

Figure 4 : variations des proportions des notes globales au cours du temps sur un échantillon mixte de 75 individus.

\section{Reproductibilité du système de notation}

Les résultats des analyses de variance effectuées sur les 76 notations simultanées des quatre notateurs ont montré que les différences n'ont pas été très significatives entre les notateurs (au seuil de 5 p. 100) surtout pour la note de dos (note de dos, $p=0,037$; note de flanc, $p=0,013$; note globale, $p=0,012$ ). Selon les couples de notateurs, les coefficients de corrélation entre notes globales, notes de dos et notes de flanc ont été respectivement compris entre $80-85$ p. 100 (tableau III), 70-80 p. 100 et 60-70 p. 100. L'accord entre les notateurs a été meilleur pour les notes arrière. Ce résultat indique que les critères d'engraissement sont plus faciles à observer en vue arrière que par le côté (observation effectuée sur d'autres espèces, notamment bovines). La moyenne des deux notes a considérablement amélioré l'accord entre les notateurs. Ce résultat découle du mode de calcul de la note globale. L'addition « note de flanc + note de dos » réduit les écarts entre les notes globales des différents notateurs et augmente leur coefficient de corrélation (supposons qu'un notateur attribue les notes 2 pour le dos et 3 pour le flanc, et que le second, sur le même animal, attribue 3 pour le dos et 2 pour le flanc, l'écart des notes entre notateurs est de 1 pour chaque position et pourtant la note globale de chaque notateur est identique, 2,5). Dans la suite du texte, la «note » désigne la note globale.

Il y a eu un accord parfait entre deux notateurs (deux notes égales) dans 56 p. 100 des cas seulement, mais dans 95 p. 100 des cas, l'écart de notes a été inférieur ou égal à 0,5 point. Si l'on compare les réponses entre trois, puis entre quatre notateurs, on constate que l'accord parfait s'est réduit respectivement à 49 et 25 p. 100 des cas. Enfin, l'harmonie n'a pas été très grande entre le quatrième notateur, moins rompu à l'utilisation de la grille, et les autres (tableau III).

\section{Tableau III}

Matrice des corrélations entre les notes globales attribuées par quatre notateurs sur 76 ânes

$\begin{array}{cccc} & \text { Notateur 1 } & \text { Notateur 2 } & \text { Notateur 3 } \\ \text { Notateur 1 } & 1 & & \\ \text { Notateur 2 } & 0,85 & 1 & \\ \text { Notateur 3 } & 0,85 & 0,83 & 1 \\ \text { Notateur 4 } & 0,79 & 0,81 & 0,77\end{array}$




\section{Effets de la saison et du sexe sur la note d'état corporel}

La figure 5 représente les variations de notes de trois groupes d'individus mâles. Dans chaque groupe, tous les individus ont partagé la même note initiale (G4m signifie groupe de mâles ayant eu la note 4 en octobre 1996). L'effectif des groupes a été de 16 individus pour $\mathrm{G} 4 \mathrm{~m}$, de 21 pour $\mathrm{G} 3 \mathrm{~m}$ et de 4 pour G2m. D'octobre à janvier, il a été constaté une baisse de la note moyenne de $\mathrm{G} 4 \mathrm{~m}$ (de 3,8 à 3,2), une stagnation de celle de G3m et une augmentation de celle de $\mathrm{G} 2 \mathrm{~m}$ (de 2 à 2,8). Octobre a marqué le passage à la saison sèche. L'adaptation au changement de saison s'est traduite par un léger amaigrissent des animaux en très bon état et par une remise en forme de ceux en mauvais état qui ont semblé tirer parti

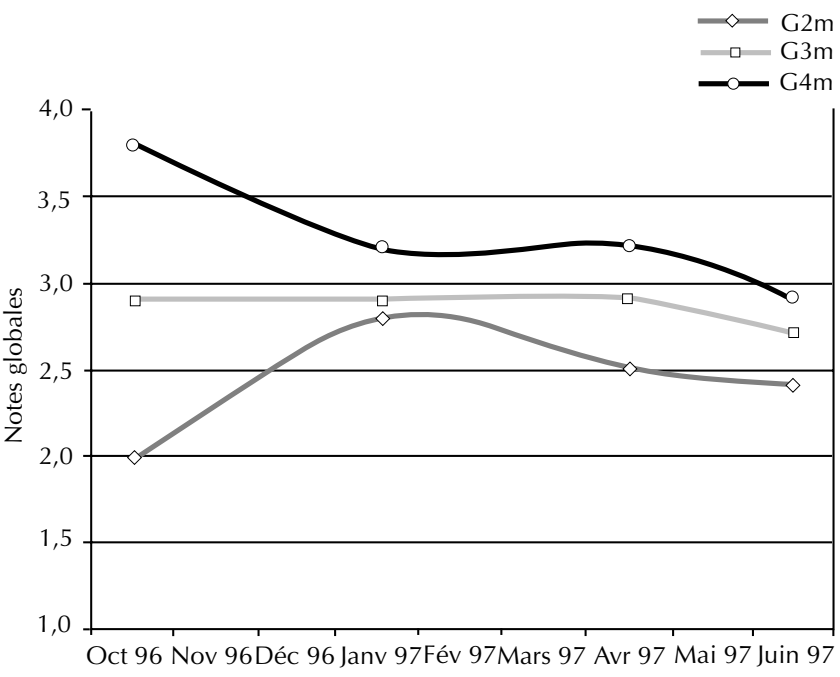

Figure 5 : variations des notes globales des ânes mâles répartis en quatre groupes selon leur note au début du suivi. $G 2 m$ (note $2 ; N=4$ individus) ; $G 3 m$ (note $3 ; N=21$ ), G4m (note $4 ; N=16)$.

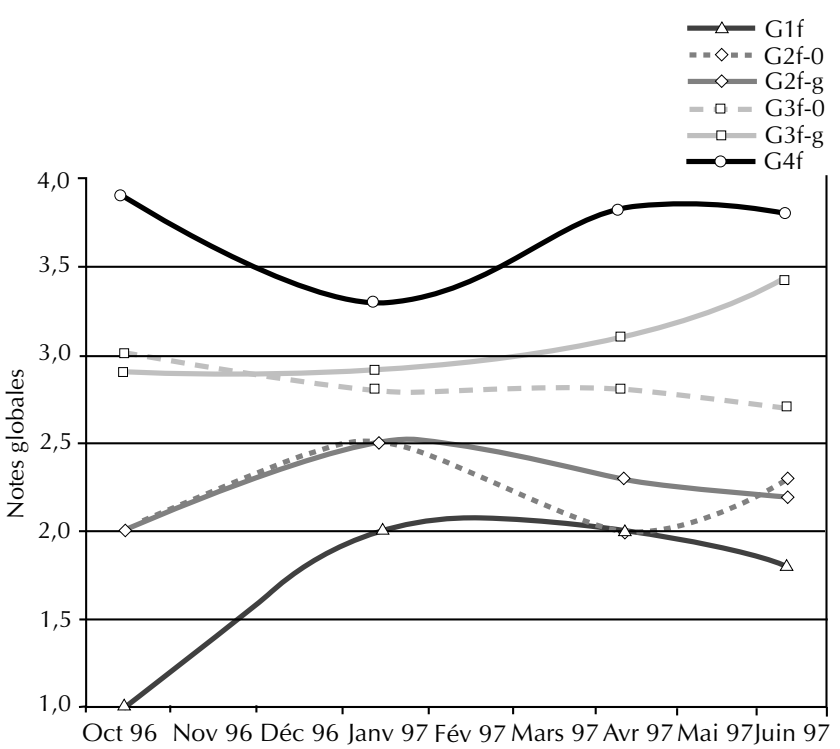

Figure 6 : variations des notes globales des ânesses réparties en quatre groupes selon leur note au début du suivi. G1f (note $1: N=2$ individus), G2f (note $2 ; N=12$ ), G3f (note $3 ; N=16$ ), G4f (note $4 ; N=4$ ). Dans les groupes 2 et 3, le suffixe g désigne les femelles gravides et le suffixe 0 les ânesses non-gravides (dans les groupes 1 et 4, l'effectif a été trop faible pour distinguer les cas). du changement d'environnement parasitaire et pathologique. A partir de janvier a été constatée une diminution lente des notes dans tous les groupes avec une accélération de la baisse à partir du mois d'avril. Cette baisse a été une conséquence directe de la raréfaction des pâturages naturels en fin de saison sèche.

La figure 6 représente les variations de notes des quatre groupes de femelles définis selon les mêmes critères que les groupes mâles. L'effectif des groupes a été de 2 pour G1f, de 12 pour G2f, de 16 pour G3f et de 4 pour G4f. Seuls les groupes G3m et G3f ont eu des effectifs relativement importants et comparables, respectivement 21 et 16. Pour cette raison, les ânesses gravides (suffixe -g sur le graphe) ont été distinguées des ânesses non-gravides (-0). Les déséquilibres et l'insuffisance des effectifs dans les trois autres cas ne pouvaient qu'entacher les analyses et les conclusions. Cette réserve étant faite, l'analyse comparative des sexes a révélé des similitudes et quelques différences aussi. Du mois d'octobre au mois de janvier, les variations de notes des groupes femelles ont été comparables à celles des groupes mâles : baisse pour G4f de 3,9 à 3,3; stagnation pour G3f au voisinage de 3 ; augmentation pour G2f de 2 à 2,5 ; et augmentation pour G1f de 1 à 2 . En revanche, de janvier à juin les variations des notes de G4f et de G3f ont divergé par rapport à celles des groupes mâles correspondants. La note de G4f a augmenté (de 3,3 à 3,8), celle de G3f-0 a été en légère diminution comme celles de $\mathrm{G} 4 \mathrm{~m}$ et de $\mathrm{G} 3 \mathrm{~m}$ sur la même période. En revanche, la note de G4f-g a été en nette augmentation de janvier à juin, car les ânesses sont arrivées en fin de gestation en fin de saison sèche. Sur la même période (janvierjuin), la note de $\mathrm{G} 2 \mathrm{f}$ comme celle de $\mathrm{G} 2 \mathrm{~m}$ a légèrement diminué avec cependant une légère remontée à partir d'avril. D'avril à juin, la divergence des notes n'a pas été nette entre les gravides et les non-gravides comme cela a été observé pour G3f (la gestation était peut-être moins avancée dans ce groupe). G1f a amorcé une nouvelle baisse à partir d'avril, franchissant le seuil de la note 2 , ce qui était inquiétant pour ces individus et leur propriétaire à l'approche de la saison des cultures. Enfin, les femelles en bon état nutritionnel ont paru plus fertiles ce qui les a dotées d'un attrait supplémentaire.

\section{DISCUSSION ET CONCLUSION}

Cette étude a permis de caractériser quatre niveaux d'état corporel pertinents pour la population des ânes du Nord Cameroun. La concordance des notes entre la grille de Pearson et Ouassat (5) et celle proposée dans cet article n'est pas parfaite. Les correspondances sont indiquées dans le tableau IV. Pearson et Ouassat font une distinction très fine entre la note 2 et la note 3 , basée sur une proéminence plus marquée des os du bassin, de la colonne vertébrale et des côtes pour la note 2 , liée à une légère différence d'épaisseur de gras et de muscle. Dans la grille de la présente étude, ces deux notes sont confondues dans la note 2 , d'où un décalage pour la suite. Finalement, on peut conclure que les ânes du Nord Cameroun se situent sur la plage comprise entre les notes 1 et 5 de la grille de ces auteurs. Bien que les statistiques concernant la barymétrie et la morphologie des ânes d'Afrique subsaharienne soient très rares, les quelques valeurs disponibles $(2,4)$ indiquent qu'ils sont d'un format comparable à celui des individus du Nord Cameroun, ce qui élargit la portée des systèmes de notation proposés.

Tout au long de la saison sèche, en moyenne 70 p. 100 des ânes du Nord Cameroun ont été dans un état corporel satisfaisant (note 3 ou 4). Cependant, cet état a différé nettement selon les sexes. Chez les mâles, les mauvaises notes (note 2) ont augmenté progressivement d'octobre à juin, passant de 10 à 25 p. 100. En revanche, pour les femelles la situation a été nettement moins bonne puisque 


\section{Tableau IV}

Concordance des notes globales entre la grille de Pearson et Ouassat ${ }^{*}$ et celle proposée dans cet article

\begin{tabular}{lccccc} 
Présente étude & Emacié & Maigre & Moyen & Bon & Gras, obèse \\
& 1 & 2 & 3 & 4 & Non observé \\
\hline \multirow{2}{*}{ Pearson et Ouassat } & Très maigre & Maigre, peu maigre & Moins que moyen & Moyen & Gras, obèse \\
& 1 & 2 et 3 & 4 & 5 & 6 à 9
\end{tabular}

*2001, Edinburgh, UK, CTVM

30 à 40 p. 100 des individus ont été en mauvais état corporel tout au long de la saison sèche. Rappelons que des prélèvements de sang effectués en octobre 1996 et janvier 1997 avaient révélé un taux d'hématocrite inférieur à 25 p. 100 chez 18 à 20 p. 100 des femelles contre 2,5 à 5 p. 100 chez les mâles (10). Tout indique que la situation des femelles ne s'améliore pas en saison des pluies, contrairement à celle des mâles, surtout lorsque pendant la lactation vient s'ajouter le travail des champs (de juin à octobre, la proportion de note 2 chez les femelles est passée de 35 à 40 p. 100, contre 25 à 10 p. 100 chez les mâles). Ces résultats montrent clairement que pour cette espèce les actions d'amélioration de l'alimentation doivent être conduites en priorité pour les femelles.

L'échantillon a certes été d'une taille très moyenne. Il est dommage de n'avoir pas poursuivi les observations en saison des pluies. Il aurait été bon de recommencer le suivi une seconde année sur un échantillon élargi pour confirmer ces résultats et affiner les dessins, notamment pour prendre en compte les spécificités des femelles. Malgré ces limites et même si un accord parfait entre deux notateurs n'a été obtenu qu'une fois sur deux environ, dans 95 p. 100 des cas la différence entre la note globale de deux notateurs a été inférieure à 0,5 point. La reproductibilité des notations a été voisine de 80 p. 100, ce qui paraît acceptable. Il semble opportun de rappeler qu'un apprentissage est toutefois nécessaire pour noter avec précision. S'il comporte une part d'imprécision non négligeable, ce système d'évaluation de l'état corporel par notation visuelle est infiniment plus facile et moins coûteux à mettre en œuvre qu'un suivi pondéral individuel. C'est un outil qui peut être facilement utilisé sur le terrain par les techniciens de l'élevage, dans une perspective d'alerte et de conseil du producteur sur la nécessité de renforcer l'alimentation de l'animal à l'approche du seuil critique de 2,5.

\section{Remerciements}

Ce travail a été réalisé grâce au concours du projet de Développement du paysannat et de gestion de terroirs (Dpgt) et du Fonds d'aide pour la coopération française $(\mathrm{Fac})$.

\section{BIBLIOGRAPHIE}

1. EBANGI A.L., VALL E., 1998. Phenotypic characterisation of draft donkeys within the Sudano-Sahelian zone of Cameroon. Revue Elev. Méd. vét. Pays trop., 51: 327-334.

2. FRICK T., 2001. Animal drawn mechanisation of field work for subsistence-based small farms in south-west Niger. Technical and interdisciplinary investigations. Stuttgart, Germany, Verlag Grauer, 142 p.

3. ITEB, 1984. Grille de notation de l'état d'engraissement des vaches pie noires. Paris, France, Iteb, $32 \mathrm{p}$.

4. LE THIEC G., 1996. Agriculture africaine et traction animale. Montpellier, France, Cirad, 355 p. (Coll. Techniques)

5. PEARSON R.A., OUASSAT M., 2001. A guide to live weight estimation and body conditions scoring of donkeys. Edinburgh, UK, CTVM, 21 p.
6. RAVENEAU A., DAVEZE J., 1997. Le livre de l'âne. Paris, France, Editions Rustica, 127 p.

7. SODECOTON, 2001. Rapport semestriel de mai à octobre 2001 Campagne agricole 01/02. Garoua, Cameroun, Sodécoton, 56 p.

8. VALL E., 1996. Capacités de travail, comportement à l'effort et réponses physiologiques du zébu, de l'âne et du cheval au NordCameroun. Thèse Doc., Ensam, Montpellier, France, 418 p.

9. VALL E., EBANGI A.L., ABAKAR O., 1996. La traction asine autour des villes de Guider, Kaélé et Mokolo au Nord-Cameroun. Rapport final d'une enquête sur I'utilisation et la conduite des ânes de trait au Nord-Cameroun. Garoua, Cameroun, Irad, Montpellier, France, Cirad-emvt, 60 p.

10. VALL E., EBANGI A.L., ABAKAR O., 1998. Culture attelée asine santé et alimentation. Rapport final. Garoua, Cameroun, Irad, 62 p.

Reçu le 04.02.2002, accepté le 03.06.2002 


\section{Summary}

Vall E., Ebangi A.L., Abakar O. Scoring Scale to Assess the Body Condition of Draft Donkeys in North Cameroon

Donkey traction began in North Cameroon in the 1970's and is today used by 25,000 smallholders. The donkey work power is just about sufficient to perform at the smallholding level. To sustain donkeys' work effort it is essential to keep them fed adequately, which is not nearly the case today. In this study the nutritional status of the draft donkey was characterized by a body condition scoring (BCS) scale. A BCS from 1-4 (emaciated, thin, average, good) was given to the back and flank of the donkey after considering by sight the pelvis, spinal column and side. The average of both scores were rounded up to the half point above to obtain the final score. The correlation of scores among various score givers was about $80 \%$, which was a good indication of its reproducibility. A total of 41 males and 34 females were monitored. A BCS of 3 prevailed (50-70\%) during the dry season. Thin animals (BCS of 2) rebuilt their food reserves in October-January, benefiting from seasonal and pathological changes. On the other hand, good animals (BCS of 4) lost weight at the first harsh occurrence of the dry season. All the animals but she-donkeys lost weight from January to June because feed resources became progressively scarce. A BCS of 2 was given in $10 \%$ of males at the beginning of the dry season and in $20 \%$ at the end of the dry season, whereas that score of 2 remained constant in $30-40 \%$ females all year round. Actions to improve the donkey nutritional status should be aimed primarily at females.

Key words: Donkey - Body condition - Evaluation Nutritional surveillance - Animal power - Cameroon.

\section{Resumen}

Vall E., Ebangi A.L., Abakar O. Realización de una escala de notación del estado corporal de los asnos de tracción

La tracción asnina, utilizada en el norte de Camerún desde los años 1970, es utilizada hoy en día por 25000 "pequeños" agricultores. La capacidad de trabajo del asno es apenas suficiente para los trabajos agrícolas. El mantenimiento de un estado nutricional correcto es determinante para garantizar una resistencia al esfuerzo aceptable. Esta condición está lejos de ser satisfecha. En el presente estudio, se caracterizó el estado nutricional de los asnos de tracción del Norte de Camerún, mediante una escala de notación del estado corporal. Se atribuyó a ojo una nota de lomo y una nota de flanco, sobre una escala de 1 a 4 (emaciado, flaco, medio, bien), según el aspecto de la pelvis, de la columna vertebral y del lado. El promedio de las dos notas, redondeado al medio punto superior, dio la nota global. La correlación de las notas entre diferentes anotadores, estuvo cercana a $80 \%$, lo que indica un buen índice de reproducibilidad. Un seguimiento de 41 machos y 34 hembras mostró que durante la estación seca, la nota 3 dominó ampliamente (50 a 70\%). Entre octubre y enero, los animales "flacos » (nota 2), habiendo beneficiado del cambio de estación y de ambiente patológico, reconstituyeron sus reservas, mientras que los animales "bien" (nota 4) adelgazaron con los primeros rigores de la estación seca. De enero a junio la escasez progresiva de los recursos alimentares provocó un adelgazamiento general, a excepción de las hembras preñadas. En los machos, la proporción de notas 2 sobrepasó de 10 a $20 \%$ entre el inicio y el final de la estación seca. En las hembras, esta proporción fue importante y constante a lo largo del año (30 a 40\%). Las acciones de mejoramiento de la alimentación deben concentrarse en las hembras.

Palabras clave: Asno - Condición corporal - Evaluación Vigilancia nutricional - Energía animal - Camerún. 